\title{
AN UNCOUNTABLE COLLECTION OF CHAINABLE CONTINUA
}

\author{
BY
}

DAVID P. BELLAMY $\left({ }^{1}\right)$

\begin{abstract}
We demonstrate the existence of an uncountable collection of chainable continua, no member of which can be mapped onto any other member. Path connectedness considerations and techniques involving compactifications play an important role in the construction and argument.
\end{abstract}

1. Introduction. In [8], J. W. Rogers, Jr., raises the question: "Does there exist an uncountable collection of chainable continua, no member of which can be mapped onto any other member?" It is the purpose of this paper to provide an affirmative answer to this question. In [9], Z. Waraszkiewicz has exhibited an uncountable family of plane continua no member of which can be mapped onto any other. Since by Theorem 4 of [4], chainable continua are planar, the construction herein provides another solution to Waraszkiewicz's problem.

We also obtain as a corollary the known result [5], [7] that there exists an uncountable collection of circularly chainable continua, no member of which can be mapped onto any other. I am indebted to the referee for indirectly pointing this out to me. Unlike those in [5], [7] the continua here are planar.

The procedure is as follows. First we describe a countable collection of chainable continua with the desired property; then we use this countable collection to construct our uncountable collection. First we need some terminology and preliminary results.

\section{Preliminaries.}

Definition 2.1. A Hausdorff compactification $S$ of a half-open interval $(a, b]$ is called a pseudocone. Let $i:(a, b] \rightarrow S$ be the injection map. $i(b)$ is the vertex of $S$, and the remainder $S-i(a, b]$ is called the base of $S$. If a space $X$ is homeomorphic to the base of $S, S$ is a pseudocone over $X$ [3].

LEMMA 2.2. If $X$ is a metric continuum, there exists a pseudocone over $X$.

References. (A proof of this result can be found in [2] or [3]. It is also a special case of a theorem in [1].)

Definition 2.3. Let $A=\left[-\frac{1}{2}, 0\right) \cup\left(0, \frac{1}{2}\right]$; and let $A(-)=\left[-\frac{1}{2}, 0\right) ; A(+)=\left(0, \frac{1}{2}\right]$. Then $A$ is just the disjoint union of two half-open intervals, $A(+)$ and $A(-)$. In

Received by the editors August 27, 1970.

AMS 1970 subject classifications. Primary 54D35, 54F20.

Key words and phrases. Chainable continuum, mappings on continua, compactification.

(1) The author was supported by the University of Delaware Research Foundation.

Copyright (C) 1971, American Mathematical Society 
keeping with the previous definition, a compactification $S$ of $A$, with injection map $i: A \rightarrow S$, will be called a pseudosuspension of its remainder $S-i(A)$ provided that every point of $S-i(A)$ is a limit point of both $i(A(-))$ and $i(A(+)) . S-i(A)$ is called the equator of $S$; and $i\left(\frac{1}{2}\right)$ and $i\left(-\frac{1}{2}\right)$ are the upper and lower vertices, respectively, of $S$.

LEMMA 2.4. There exists a pseudosuspension of any metric continuum and any such pseudosuspension is metrizable.

Proof. To obtain a pseudosuspension of $X$, start with the disjoint union of two pseudocones over $X$ and identify corresponding points in the bases.

Suppose $S=A \cup X$ is a pseudosuspension of $X$. Here, for simplicity, we take $A$ and $X$ to be subsets of $S$. Let $f: X \rightarrow C$ be an embedding, where $C$ is again the Hilbert cube. Let $g: S \rightarrow C$ be a continuous map extending $f$. This exists since $C$ is an absolute retract. Define $h: S \rightarrow C \times\left[-\frac{1}{2}, \frac{1}{2}\right]$ by

$$
\begin{aligned}
& h(x)=(g(x), x) \text { for } x \in A \text {, } \\
& =(g(x), 0) \text { for } x \in X .
\end{aligned}
$$

Then $h$ is an embedding of $S$ into a metric space.

The next result is stated without proof. It follows from standard techniques used with such spaces as the usual topologist's $\sin 1 / x$ curve.

LEMMA 2.5. The set of path components of a pseudosuspension of a nondegenerate space $X$ consists of the collection of path components of $X$ together with the two sets $i(A(+))$ and $i(A(-))$.

Notation. If $S$ is a metric space and $x \in S$, let $V(x, \varepsilon)=\{y: d(x, y)<\varepsilon\}$.

The next result is perhaps obvious. It is included because the traditional way of considering continua has been in the context of some underlying embedding space, while for our purpose here we are considering them as spaces in their own right without reference to any larger space containing them (except as may be convenient for particular constructions). The lemma allows us a certain freedom in the construction of chains later.

LEMMA 2.6. A metric continuum $X$ is chainable if and only if, given any metric space $S$ in which $X$ is embedded and any $\varepsilon>0$, there is an $\varepsilon$-chain $\left\{U_{i}\right\}_{i=1}^{n}$ of open subsets of $S$ such that each $U_{i}$ meets $X$ and $X \subseteq \bigcup_{i=1}^{n} U_{i}$.

LEMMA 2.7. A pseudosuspension $S$ of a chainable continuum $X$ is itself a chainable continuum; in fact, its vertices are opposite endpoints.

Indication of proof. The proof of this result is tedious but straightforward. One first chains $X$ with open subsets of $S$, then chains appropriate subarcs of $A(+)$ and $A(-)$, and finally discards enough of each link of the chain on $X$ to make the entire cover a chain of $S$. 
LEMMA 2.8. If $S$ and $Z$ are pseudosuspensions of nondegenerate spaces $X$ and $Y$, $X \subseteq S$ and $Y \subseteq Z$, and $f: S \rightarrow Z$ is continuous and onto, then $f(X)=Y$.

Proof. Let $i: A \rightarrow S$ and $j: A \rightarrow Z$ be the injection maps. Then $f(i(A))$ is dense in $Z$. In particular, $f(i(A)) \cap j(A(+)) \neq \varnothing$, since $j(A(+))$ is open in $Z$. Let $t \in f(i(A)) \cap j(A(+))$. Then either $t \in f(i(A(+)))$ or $t \in f(i(A(-)))$; by symmetry we may suppose $t \in f(i(A(+)))$. Then since $f(i(A(+)))$ is path connected and meets $j(A(+))$, a path component of $Z$, it follows that $f(i(A(+))) \subseteq j(A(+))$. But since there is some $s \in f(i(A)) \cap j(A(-))$, also, it follows that $s \in f(i(A(-)))$ and that $f(i(A(-))) \subseteq j(A(-))$. Thus, $f(i(A)) \subseteq j(A)$, so that

$$
f(X)=f(S-i(A)) \supseteq Z-j(A) \supseteq Y .
$$

To show that $f(X) \subseteq Y$ and complete the proof, suppose $y \in f(X)$. If $y \in j(A(+))$, $f^{-1}(j(A(+)))$ is an open subset of $S$ which contains some point $x$ of $X$, since $y \in f(X)$. Thus, since $x$ is a limit point of $i(A(-))$, it follows that

$$
f^{-1}(j(A(+))) \cap i(A(-)) \neq \varnothing .
$$

Then, $f\left(f^{-1}(j(A(+))) \cap i(A(-))\right) \neq \varnothing$ so that $j(A(+)) \cap f(i(A(-))) \neq \varnothing$, a contradiction, since $f(i(A(-))) \subseteq j(A(-))$. Similarly we cannot have $y \in j(A(-))$. Therefore, $y \in Y$ and $f(X)=Y$ as desired.

3. The countable collection. First define $M_{0}$ as follows: Let

$$
\begin{aligned}
& B(+)=\left\{(x, y) \in E^{2}: 0<x<1 \text { and } y=\sin \left(x^{3}-x\right)^{-1}\right\}, \\
& B(-)=\left\{(x, y) \in E^{2}:-1<x<0 \text { and } y=\sin \left(x^{3}-x\right)^{-1}\right\} .
\end{aligned}
$$

[The sole important feature of the $x^{3}-x$ here is that this is a continuous function with zeros at $-1,0$, and 1 , only.] For $i=-1,0,1$, set

$$
C_{i}=\left\{(i, y) \in E^{2}:-1 \leqq y \leqq 1\right\} .
$$

Then set $M_{0}=B(+) \cup B(-) \cup \cup_{i=-1}^{1} C_{i}$. It is clear that $M_{0}$ is a chainable continuum; it is atriodic and unicoherent and [6, Corollary 1, p. 467] applies, since it is a union of four topological copies of the usual $\sin 1 / x$ curve. Now, proceed resursively: If $M_{k}$ has been chosen, let $M_{k+1}$ be any pseudosuspension of $M_{k}$. One always exists by Lemma 2.4. We thus obtain a collection $\left\{M_{k}\right\}_{k=0}^{\infty}$ of continua. For $k>0, M_{k}$ is a pseudosuspension of $M_{k-1}$. For each $k \neq 0$, let $N_{k} \subseteq M_{k}$ be the equator; that is, $N_{k}$ is the copy of $M_{k-1}$ in $M_{k}$.

Proposition 3.1. Each $M_{k}$ is chainable.

Proof. $M_{0}$ is chainable. If $M_{k}$ has been shown to be chainable, $M_{k+1}$ is chainable by Lemma 2.7 . We are done by induction.

Proposition 3.2. If $k<l$, there is no continuous onto mapping $f: M_{k} \rightarrow M_{l}$.

Proof. $M_{k}$ has fewer path components than $M_{l}$ in this case. 
Lemma 3.3. No pseudosuspension $S$ can be mapped onto $M_{0}$.

Proof. Suppose $S=i(A) \cup X$. Let $B(+) \cup B(-)=B$. If $f: S \rightarrow M_{0}$ is onto then $f(i(A))$ is dense in $M_{0}$ and has at most two path components. The only possibility, then, is that $f(i(A))=B$. Since $B$ has two path components, separate path components of $i(A)$ must be mapped onto separate path components of $B$; we may by symmetry assume that $f(i(A(+)))=B(+)$ and $f(i(A(-)))=B(-)$. Then let $p \in f^{-1}\left(C_{-1}\right) . p \in X$ since it cannot be in $i(A) . C_{-1} \cup B(-)$ is open in $M_{0}$, and contains $f(p)$, so that $f^{-1}\left(C_{-1} \cup B(-)\right)$ is open in $S$ and contains $p$. Since $p \in X$, $f^{-1}\left(C_{-1} \cup B(-)\right) \cap i(A(+)) \neq \varnothing$, or $\left(C_{-1} \cup B(-)\right) \cap f(i(A(+))) \neq \varnothing$, a contradiction since $f(i(A(+)))=B(+)$, which does not meet $C_{-1} \cup B(-)$.

COROllaRy 3.4. There is no continuous map from $M_{k}$ onto $M_{0}$ unless $k=0$.

Proof. For $k \neq 0, M_{k}$ is a pseudosuspension.

Proposition 3.5. If $k>l$ there is no continuous onto mapping $f: M_{k} \rightarrow M_{l}$.

Proof. We argue by induction on $l$. For $l=0$, this is the preceding corollary. Assume we have shown that there is no onto mapping $f: M_{k} \rightarrow M_{n}$ for $k>n$. Suppose $k>n+1$ and $f: M_{k} \rightarrow M_{n+1}$ is onto and continuous. Then, by Lemma 2.8, $f \mid N_{k}$ maps $N_{k}$ onto $N_{n+1}$. Let $h: N_{k} \rightarrow M_{k-1}$ and $g: N_{n+1} \rightarrow M_{n}$ be homeomorphisms. Then $g f h^{-1}: M_{k-1} \rightarrow M_{n}$ is a continuous surjection. Since $k-1>n$, this contradicts the induction hypothesis and completes the proof.

Combining Propositions 3.2 and 3.5, we have

THEOREM 3.6. If $k \neq l, M_{k}$ cannot be mapped onto $M_{l}$.

4. The uncountable collection. Let $Z^{+}$denote the positive integers. The uncountability of the collection to be constructed depends on the following result. The symbol $\mathscr{A}$ will henceforth denote the set described here.

LEMMA 4.1. There is a collection $\mathscr{A}$, with cardinal number $c$, of infinite subsets of $Z^{+}$, with the property that no member of $\mathscr{A}$ is a subset of any other member of $\mathscr{A}$.

Proof. Let $Q$ denote the rationals and let $\eta: Z^{+} \rightarrow Q$ be $1-1$ and onto. For each real number $t$, let $J_{t}=\eta^{-1}(t, t+1)$. By density of the rationals, distinct values of $t$ yield distinct sets $J_{t}$. It is clear that no $J_{t}$ is contained in any other. Let $\mathscr{A}=\left\{J_{t}\right\}_{t \in E^{1}}$.

Construction of THE Example 4.2. (The reader is referred to the Appendix for a more intuitive description.) For each $J \in \mathscr{A}$ ( $\mathscr{A}$ as above), construct the continuum $W(J)$ as follows: First, let $J=\left\{a_{i}\right\}_{i=1}^{\infty}$. The indexing could be arbitrarily chosen, but for simplicity we choose the unique indexing for which $a_{i+1}>a_{i}$ for every $i$; that is, $\left\langle a_{i}\right\rangle_{i=1}^{\infty}$ is a strictly increasing sequence of integers whose range is $J$.

Now, for each $i \in Z^{+}$, let $\mu_{i}: A \rightarrow M_{i}$ be a homeomorphism of $A$ onto the dense copy of $A$ in $M_{i}$. Then, let $X(J)$ denote the free disjoint union of $\left\{M_{j}\right\}_{j \in J}$. Let $\hat{X}(J)=X(J) \cup\left\{\omega_{J}\right\}$ denote the one point compactification of $X(J) . W(J)$ is then 
the continuum obtained from $\hat{X}(J)$ by identifying $\mu_{a_{i}}\left(+\frac{1}{2}\right)$ with $\mu_{a_{i+1}}\left(-\frac{1}{2}\right)$ for each positive integer $i$. That is, we identify the upper vertex of each $M_{a_{i}}$ with the lower vertex of the next one.

We need to introduce inclusion maps $\varphi(J, j): M_{j} \rightarrow W(J)$ for bookkeeping purposes. This inclusion map is defined for each pair $(J, j)$ where $J \in \mathscr{A}$ and $j \in J$, since these are the only cases for which a copy of $M_{j}$ (with nonempty interior) is contained in $W(J)$.

Proposition 4.3. Each $W(J)$ is chainable.

Proof. Let $d$ be a metric on $W(J)$ and let $\varepsilon>0$ be given. Then there is an $n$ such that

$$
\operatorname{dia}\left(W(J)-\bigcup_{i=1}^{n} \varphi\left(J, a_{i}\right)\left(M_{a_{i}}\right)\right)<\varepsilon .
$$

Let $U_{0}=W(J)-\bigcup_{i=1}^{n} \varphi\left(J, a_{i}\right)\left(M_{a_{i}}\right)$. Then the complement of $U_{0}$ is a finite union of copies of $M_{j}$ 's with the upper vertex of each (except the last) joined to the lower vertex of the next. Since each $M_{j}$ is chainable, it is straightforward to verify directly that $W(J)-U_{0}$ is chainable from the upper vertex of $\varphi\left(J, a_{n}\right)\left(M_{a_{n}}\right)$ to the lower vertex of $\varphi\left(J, a_{1}\right)\left(M_{a_{1}}\right)$; that is, from $\varphi\left(J, a_{n}\right)\left(\mu_{a_{n}}\left(\frac{1}{2}\right)\right)$ to $\varphi\left(J, a_{1}\right)\left(\mu_{a_{1}}\left(-\frac{1}{2}\right)\right)$. Hence, there exists, by Lemma 2.6, an $\varepsilon$-chain $\left\{U_{i}^{\prime}\right\}_{i=1}^{m}$ of open subsets of $W(J)$ covering $\bigcup_{i=1}^{n} \varphi\left(J, a_{i}\right)\left(M_{a_{i}}\right)$. Define $U_{1}=U_{1}^{\prime}$, and for $i>1, U_{i}=U_{i}^{\prime}-\mathrm{Cl}\left(U_{0}\right)$. Then $\left\{U_{i}\right\}_{i=0}^{m}$ is an $\varepsilon$-chain covering $W(J)$, and the proof is complete.

The next result will complete the argument.

THEOREM 4.4. If $J=\left\{a_{i}\right\}_{i=1}^{\infty}$ and $K=\left\{b_{i}\right\}_{i=1}^{\infty}$ are members of $\mathscr{A}$, and there exists a continuous surjection $f: W(J) \rightarrow W(K)$, then $W(J)=W(K)$.

Proof. We need only show that $J=K$, and by the properties of $\mathscr{A}$ given in Lemma 4.1, it suffices to show that $K \subseteq J$. We first establish a lemma.

LEMMA 4.5. $f\left(\omega_{J}\right)=\omega_{K}$.

Proof of lemma. First suppose $p \in W(J), p \neq \omega_{J}$, and that $f(p)=\omega_{K}$. Let $q$ be any point in $W(J)-\left\{\omega_{J}\right\}$. Then, for some integer $n$, both $p$ and $q$ lie in $L_{n}$, where

$$
L_{n}=\bigcup_{i=1}^{n} \varphi\left(J, a_{i}\right)\left(M_{a_{i}}\right)
$$

$L_{n}$, then, is a continuum with only finitely many path components, since each $M_{a_{i}}$ has only finitely many. Hence, $f\left(L_{n}\right)$ is also a continuum with only finitely many path components, and containing $\omega_{K}$ since $f(p)=\omega_{K}$. Since every nondegenerate subcontinuum of $W(K)$ containing $\omega_{K}$ has infinitely many path components (it contains, for example, $\varphi\left(K, b_{i}\right)\left(N_{b_{i}}\right)$ for infinitely many values of $i$; and each of these continua is a union of path components of $W(K))$, it follows that $f\left(L_{n}\right)$ $=\left\{\omega_{K}\right\}$. Thus, in particular $f(q)=\omega_{K}$, and since $q$ was arbitrary, $f\left(W(J)-\left\{\omega_{J}\right\}\right)$ 
$=\left\{\omega_{K}\right\}$. By continuity, $f\left(\omega_{J}\right)=\omega_{K}$, also, and $f$ is not onto, a contradiction. Hence, for $p \neq \omega_{J}, f(p)$ cannot equal $\omega_{K}$. Since $f$ is onto, it must map some point to $\omega_{K}$, and the only remaining possibility is that $f\left(\omega_{J}\right)=\omega_{K}$.

Returning to the proof of Theorem 4.4, we introduce the following notation: $\left[\frac{1}{2}, \infty\right]$ denotes the one point compactification of the half-open interval $\left[\frac{1}{2}, \infty\right)$, with $\infty$ as point of compactification.

Define $\Pi_{J}: W(J) \rightarrow\left[\frac{1}{2}, \infty\right]$ by

$$
\begin{aligned}
& \Pi_{J}\left(\omega_{J}\right)=\infty, \\
& \Pi_{J}\left(\varphi\left(J, a_{i}\right)\left(\mu_{a_{i}}(t)\right)\right)=t+i, \\
& \Pi_{J}(x)=i \text { if } x \in \varphi\left(J, a_{i}\right)\left(N_{a_{\imath}}\right) .
\end{aligned}
$$

Then $\Pi_{J}$ is continuous, monotone and onto, and $\Pi_{J}^{-1}(t)$ is a single point unless $t$ is an integer, in which case $\Pi_{J}^{-1}(t)=\varphi\left(J, a_{t}\right)\left(N_{a_{t}}\right)$. Define $\Pi_{K}: W(K) \rightarrow\left[\frac{1}{2}, \infty\right]$ in precisely the same fashion, merely replacing $J$ by $K$ and $a_{i}$ by $b_{i}$ wherever they occur in the definition of $\Pi_{J}$.

Now, consider the point $\Pi_{K}^{-1}\left(\frac{1}{2}\right)$ in $W(K) \cdot f^{-1}\left(\Pi_{K}^{-1}\left(\frac{1}{2}\right)\right)$ is a nonempty closed subset of $W(J)$ missing $\omega_{J}$. Let $s$ be any point in $\Pi_{J}\left(f^{-1}\left(\Pi_{K}^{-1}\left(\frac{1}{2}\right)\right)\right)$. Then $s \neq \infty$, and since $\Pi_{J}$ is montone, $f\left(\Pi_{J}^{-1}[s, \infty]\right)$ is a subcontinuum of $W(K)$ containing both $\Pi_{K}^{-1}\left(\frac{1}{2}\right)$ and $\omega_{K}$. Since $W(K)$ is irreducible between these two points,

$$
f\left(\Pi_{J}^{-1}[s, \infty]\right)=W(K) .
$$

Now, let $b_{i} \in K$ be arbitrary. Consider $x \in[s, \infty]$, given by $x=$ glb $V$, where

$$
V=\left\{t>s: f\left(\Pi_{J}^{-1}[s, t]\right) \cap \Pi_{K}^{-1}(i, \infty] \neq \varnothing\right\} .
$$

Then $x$ is finite, since, for example, there is a $t, \infty>t>s$, for which some point of $\Pi_{J}^{-1}(t)$ is mapped by $f$ into the set $\varphi\left(K, b_{i+1}\right)\left(N_{b_{i+1}}\right)=\Pi_{K}^{-1}(i+1)$.

We then wish to show that $f\left(\Pi_{J}^{-1}(x)\right)=\Pi_{K}^{-1}(i)$. First,

$$
f\left(\Pi_{J}^{-1}[s, x]\right) \cap \Pi_{K}^{-1}(i, \infty]=\varnothing,
$$

for suppose $y \in f\left(\Pi_{J}^{-1}[s, x]\right) \cap \Pi_{K}^{-1}(i, \infty]$. Then since $\Pi_{K}(y)>i$ and $\Pi_{K} f \Pi_{J}^{-1}[s, x]$ is a closed connected set containing both $\Pi_{K}(y)$ and $\frac{1}{2}, \Pi_{K} f \Pi_{J}^{-1}[s, x]$ contains the open interval $\left(i, \Pi_{K}(y)\right)$. Then,

$$
\Pi_{J}^{-1}[s, x] \cap f^{-1} \Pi_{K}^{-1}\left(i, \Pi_{K}(y)\right)
$$

is nonempty and is open in $\Pi_{J}^{-1}[s, x]$. Hence, since $\Pi_{J}^{-1}(x)$ has empty interior, there is an $r<x$ such that

$$
\Pi_{J}^{-1}(r) \cap \Pi_{J}^{-1}[s, x] \cap f^{-1}\left(\Pi_{K}^{-1}\left(i, \Pi_{K}(y)\right)\right) \neq \varnothing
$$

or simply

$$
\left.\Pi_{J}^{-1}(r) \cap f^{-1} \Pi_{K}^{-1}\left(i, \Pi_{K}(y)\right)\right) \neq \varnothing .
$$


Then,

$$
f\left(\Pi_{J}^{-1}(r) \cap f^{-1} \Pi_{K}^{-1}\left(i, \Pi_{K}(y)\right)\right) \neq \varnothing
$$

and

$$
f \Pi_{J}^{-1}(r) \cap \Pi_{K}^{-1}\left(i, \Pi_{K}(y)\right) \neq \varnothing,
$$

and since $\{r\} \subseteq[s, r]$ and $\left(i, \Pi_{K}(y)\right) \subseteq(i, \infty]$,

$$
f\left(\Pi_{J}^{-1}[s, r]\right) \cap \Pi_{K}^{-1}(i, \infty] \neq \varnothing,
$$

so that $r \in V$. This is a contradiction since $r<x$. Thus (i) is established, or $f \Pi_{J}^{-1}(x)$ $\subseteq \Pi_{K}^{-1}\left[\frac{1}{2}, i\right]$.

We show next that $f\left(\Pi_{J}^{-1}(x)\right) \subseteq \Pi_{K}^{-1}[i, \infty]$. Suppose $y \in f \Pi_{J}^{-1}(x) \cap \Pi_{K}^{-1}\left[\frac{1}{2}, i\right)$. Let $y_{0} \in \Pi_{J}^{-1}(x)$ be a point such that $f\left(y_{0}\right)=y$. Then $y_{0} \in f^{-1} \Pi_{K_{0}}^{-1}\left[\frac{1}{2}, i\right)$, and $f^{-1}\left(\Pi_{K}^{-1}\left[\frac{1}{2}, i\right)\right)$ is open in $W(J)$, so that

$$
f^{-1} \Pi_{K}^{-1}\left[\frac{1}{2}, i\right) \cap \Pi_{J}^{-1}(x, x+1) \neq \varnothing,
$$

since $\Pi_{J}^{-1}(x) \subseteq \mathrm{Cl}\left(\Pi_{J}^{-1}(x, x+1)\right)$. Now, $x$ is an integer, for if not there is an $\varepsilon>0$ such that $[x-\varepsilon, x+\varepsilon]$ contains no integer. Then $\Pi_{J}^{-1}[x-\varepsilon, x+\varepsilon]$ is topologically an arc, and $f \Pi_{J}^{-1}[x-\varepsilon, x+\varepsilon]$ is a path-connected continuum containing two points $y_{1}$ and $y_{2}$ with $\Pi_{K}\left(y_{1}\right) \leqq i$ and $\Pi_{K}\left(y_{2}\right)>i$. Since any such continuum meets both $\varphi\left(K, b_{i}\right)\left(N_{b_{i}}\right)$ and $\varphi\left(K, b_{i}\right)\left(\mu_{b_{i}}(A(+))\right)$, it has more than one path component, a contradiction.

Since $x$ is an integer, $\Pi_{J}^{-1}(x, x+1)$ is a path connected set, as is $f \Pi_{J}^{-1}(x, x+1)$. Since by definition of $x$ and $V, f \Pi_{J}^{-1}(x, x+1)$ meets $\Pi_{K}^{-1}(i, \infty]$, and $\Pi_{K}^{-1}(i, \infty]$ is a union of path components of $W(K)$, it follows that $f \Pi_{J}^{-1}(x, x+1) \subseteq \Pi_{K}^{-1}(i, \infty]$. This contradicts (ii). Hence $f \Pi_{J}^{-1}(x) \subseteq \Pi_{K}^{-1}[i, \infty]$, and combining this with (i), we have $f \Pi_{J}^{-1}(x) \subseteq \Pi_{K}^{-1}(i)$.

We need next to show that $\Pi_{K}^{-1}(i) \subseteq f \Pi_{J}^{-1}(x)$. For each positive $\delta, f \Pi_{J}^{-1}[x, x+\delta]$ is a continuum containing $\Pi_{K}^{-1}(i+\varepsilon)$ for some $\varepsilon>0$, and also meeting $\Pi_{K}^{-1}(i)$. We may assume $\varepsilon<1$; then $\Pi_{K}^{-1}(i, i+\varepsilon)$ is topologically an open interval, and $\Pi_{K}^{-1}(i, i+\varepsilon) \subseteq f \Pi_{J}^{-1}[x, x+\delta]$. Since $f \Pi_{J}^{-1}[x, x+\delta]$ is closed,

$$
\mathrm{Cl} \Pi_{K}^{-1}(i, i+\varepsilon) \subseteq f \Pi_{J}^{-1}[x, x+\delta]
$$

or

$$
\Pi_{K}^{-1}[i, i+\varepsilon] \subseteq f \Pi_{J}^{-1}[x, x+\delta] .
$$

In particular, then, $\Pi_{K}^{-1}(i) \subseteq f \Pi_{J}^{-1}[x, x+\delta]$. Since this holds for all $\delta>0$,

$$
\Pi_{K}^{-1}(i) \subseteq \bigcap_{\delta>0} f \Pi_{J}^{-1}[x, x+\delta]=f \Pi_{J}^{-1}(x) .
$$

Thus, $f \Pi_{J}^{-1}(x)=\Pi_{K}^{-1}(i)$ or since $x$ and $i$ are integers

$$
f\left(\varphi\left(J, a_{x}\right)\left(N_{a_{x}}\right)\right)=\varphi\left(K, b_{i}\right)\left(N_{b_{i}}\right) .
$$

But the $\varphi$ 's are embeddings, and $N_{a_{x}}$ can be mapped onto $N_{b_{i}}$ if and only if $b_{i}=a_{x}$ 
(because $N_{j}$ is homeomorphic to $M_{j-1}$ ). Since $a_{x} \in J, b_{i} \in J$ also, and since $b_{i} \in K$ was arbitrary, $K \subseteq J$ and the proof is done.

COROllaRy 4.6. There exists an uncountable collection $\{F(J): J \in \mathscr{A}\}$ of planar circularly chainable continua, no member of which can be mapped onto any other.

Proof. For each $J \in \mathscr{A}$, let $E(J)$ be a pseudosuspension of $W(J) . E(J)$ is chainable by Lemma 2.7, and planar by Theorem 4 of [4]. $F(J)$ is obtained from $E(J)$ by identifying the two vertices to a single point. $F(J)$ is then clearly circularly chainable and planar. If $f: F(J) \rightarrow F(K)$ is continuous and onto, an argument almost identical to the proof of Lemma 2.8 yields $f(W(J))=W(K)$, which implies $W(J)=W(K)$ by Theorem 4.4, and in turn $F(J)=F(K)$.

5. Appendix. A geometric description of the $W(J)$ 's. This description is perhaps more intuitive but is less suited for our arguments. Let $D_{n} \subseteq E^{2}$ denote the closed circular disc of radius $\frac{1}{2}(1 / n-1 /(n+1))$ and center $\left(\frac{1}{2}(1 / n+1 /(n+1)), 0\right)$. Then $\left\langle D_{n}\right\rangle_{n=1}^{\infty}$ is a sequence of discs, each tangent to the ones to either side, adjacent to it, converging to the origin. For each $a_{n} \in J$, let $h_{n}: M_{\alpha_{n}} \rightarrow D_{n}$ be an embedding such that $h_{n}$ takes the lower vertex of $M_{a_{n}}$ to $(1 / n, 0)$ and the upper one to $(1 /(n+1), 0)$. Such a map exists by Theorem 4 of [4]. Then set $W(J)=\{(0,0)\}$ $\cup \bigcup_{n=1}^{\infty} h_{n}\left(M_{a_{n}}\right)$.

\section{REFERENCES}

1. J. M. Aarts and P. van Emde Boas, Continua as remainders in compact extensions, Nieuw Arch. Wisk. (3) 15 (1967), 34-37. MR 35 \#4885.

2. D. P. Bellamy, A non-metric indecomposable continuum, Duke Math. J. 38 (1971), 15-20.

3. - Topological properties of compactifications of a half-open interval, Ph.D. Thesis, Michigan State University, E. Lansing, Mich., 1968.

4. R. H. Bing, Snake-like continua, Duke Math. J. 18 (1951), 653-663. MR 13, 265.

5. H. Cook, Upper semi-continuous continuum-valued mappings onto circle-like continua, Fund. Math. 60 (1967), 233-239. MR 36 \#3321.

6. J. B. Fugate, Decomposable chainable continua, Trans. Amer. Math. Soc. 123 (1966), 460-468. MR 33 \#4906.

7. J. W. Rogers, Jr., Pseudo-circles and universal circularly chainable continua, Doctoral Dissertation, University of California, Riverside, Calif., 1968.

8. - Continuous mappings on continua, Proc. Auburn Topology Conference, March 1969, pp. 94-97.

9. Z. Waraszkiewicz, Une famille indenombrable de continus plans dont aucun n'est l'image continue d'un autre, Fund. Math. 18 (1932), 118-137.

University of Delaware,

Newark, Delaware 19711 